\title{
Basic Contact and Exchange Mechanism of College Students of All Ethnic Groups
}

\author{
Wenzhang Bi \\ School of Ethnology and Sociology, Southwest Minzu University, Chengdu, China \\ Email: 80489141@qq.com
}

How to cite this paper: Bi, W.Z. (2021) Basic Contact and Exchange Mechanism of College Students of All Ethnic Groups. Open Access Library Journal, 8: e7910. https://doi.org/10.4236/oalib.1107910

Received: August 31, 2021

Accepted: September 26, 2021

Published: September 29, 2021

Copyright $\odot 2021$ by author(s) and Open Access Library Inc.

This work is licensed under the Creative Commons Attribution International License (CC BY 4.0).

http://creativecommons.org/licenses/by/4.0/

\section{(c) (i) Open Access}

\begin{abstract}
The mechanism of ethnic communication refers to the inherent regularity of ethnic communication and the internal and external social and environmental factors needed. In a social environment such as multi-ethnic universities, the establishment of a mechanism of ethnic communication according to the law needs three bases: first, the promotion of communication needs to be guided conveniently, and the resources are fine and silent. The second is to realize that ethnic exchanges are cultural exchanges between individual ethnic groups, without the need to deliberately emphasize national identity. Finally, it is necessary to create an external social environment that promotes the social mobility of individuals.
\end{abstract}

\section{Subject Areas}

Culture, Sociology

\section{Keywords}

Ethnic Groups, Exchange, Mechanism, University

\section{1. 促进民族交往的原则：春风化雨}

党的十八以来的民族工作会议, 都在强调促进民族交往交流交融[1]。但 这种促进最好的方式, 是自然而然地引导, 使得各个民族自如地交往交流。 或者说, 不是为了交流而交流。最现实的符合人们交流习惯的方式, 是通过 建立一些交往的机会或平台, 比如共同工作、学习、游戏、旅游等, 在这种 共同的行动中，实现了自然的交流，从而实现了民族团结[2]。

民族交流应该建立符合人性的自然的交往方式。现实生活中, 我们也应 尽可能避免强推式的民族交往。我们可以脑补这样的画面, 假如学校组织不 同民族的同学坐在一起交流, 同学相互间可能会产生衁尬。我国在新疆的访 
惠聚活动[3], 从 2014 年开始, 干部下乡。这种群众路线运动, 有促进民族交 往交流的目的和功能, 已经有了大量的经验总结值得我们深思 [4]。

就民族交往而言，应尽可能多地建立和创造民族交往的机会和平台。在 这些交往平台之上，各个民族在日常生活中自然而然地产生接触。接触发生 在润物细无声之处，潜移默化之中。

多民族大学可作为民族交往的平台，但大学绝不是为民族交往而建。全 国各地的莘莘学子们来到这里, 主要任务是为了学习。人际交往, 民族交往 都是附带的。但学生们想到的不仅仅是学习, 学习是主业, 但人际交往依然 很重要。十八九岁, 青春懵懂、意气奋发, 对来自五湖四海、不同文化, 甚 至不同长相的同学, 自然充满好奇。学习的同时, 也在进行各种人际交往。 甚至有学生把学习当成副业, 而把兼职、人际交往、恋爱、体验都市生活当 成了主业。大学里人际交往还是比较重要 [5]。知乎高赞里有提问是大学里学 习重要还是人际关系重要 [6]。

\section{2. 重文化交往而非民族身份交往}

人们习惯于从某个方面将人划归为某一类。男人、女人是一种划分，民 族也是一种划分。区别在于，男女性主要根据生理结构划分; 民族主要以文 化差异来划分。当然，民族间的划分相对复杂，可能还包含人种差异。只是 现代社会, 种族差异基本被忽视掉了。

从民族方面来说, 文化的差异才是真的差异。在中国, 我们知道存在自 小在上海长大, 说的一口上海话的黑人; 有在北京长大, 说的一口京腔的外 国人、哈萨克人; 有在成都长大, 说的一口地道成都话的维吾尔人、藏族人。 他们和周围的人只是长得不一样，但语言、文化、思维方式、生活习俗几乎 没有任何差别。如果长相又比较像的话，他人很容易想当然地认为他是土生 土长的本地人, 比如有些在内地出生长大的藏族人、蒙古族人、哈萨克人、 维吾尔人等就是如此。这些人和周边的人交流完全不成问题, 皆是文化差异 比较小的因素。如果把影响民族间人际交往的因素按照重要性排列出来的话, 按照常人的思维, 首先就应该是语言, 其次是宗教及生活习俗、思维观念、 外在形象等差异性了。

所以，民族间交流，首先应当具备交流的工具。其次是有能够持续交流 下去的共同点，如三观是否一致。再其次，可能才是生活习俗的差异性。最 后才可能是什么颜值。如果放在个体层面来看, “始于颜值, 终于人品”, 这样的事情经常发生。但基本前提是有共同交流的语言。语言是民族文化的 根本，是民族认同和国家认同的根本。不同民族语言的个体必须具备共通的 语言才可进行交往交流，对我国来说，国家通用语言的重要性就凸显出来[7]。 除了语言，附着于民族之上的宗教、生活习俗等在民族交往的过程中逐渐会 呈现出来。我们关注的是，两个文化差异较大的民族个体，进行正常的交流 交往需要具备什么条件。

从访谈结果发现，以民族身份来看待人际交往，民族间矛盾往往是文化 矛盾, 而非民族矛盾。民族的差异可能有冲突, 也可能很快消解掉，有的还 会成为好朋友。同一民族的反而可能相处不好。印象最深的有个维吾尔族女 
生, 她初中到高中都住校。从她的言谈中, 根本感觉不到维汉之间的差异。 她从小上汉校, 维语也懂, 和汉族同学一起长大。她说的一口典型的新疆普 通话, 不看本人, 根本区分不出来她是汉族还是维吾尔族。她思维很活跃, 总是微笑着的, 问她关于民族交往存在的问题, 她总是能够尽可能地从非民 族问题意识的方式来看待同学之间的相处。

我作为研究者则是恰恰相反。和我一起做访谈的小伙伴说, 我总是试图 将个体之间的矛盾和问题用民族的变量因素来解释, 并且我总是喜欢引导被 访者, 让对方也认为这是民族间的问题。我这种思维显得不够客观理性, 缺 乏社会学应该具有的价值中立观念。而且在现实中, 也确实没有那么多我以 为存在的民族问题。随着资料整理的逐渐增多, 我也慢慢地发现了我当初存 在的固化思维方式。如果说从民族视角可以发现一些有意义的结果, 那么跨 文化应该是一个更好的视角, 民族本质就是文化。

所以, 以民族区分人类个体, 将其类属化, 自然以民族视角分析人际交 往。然而, 这种研究视角容易忽视人际交往的共同性, 也容易强调人与人的 差异性。民族是文化的载体, 民族不是问题, 文化差异才是问题。民族交往 更多是跨文化交往，谨防文化问题民族化。

\section{3. 民族交往的个体基础：走出去}

民族大学中, 有维吾尔族、藏族、蒙古族、彝族等。这些少数民族学生, 其文化和主体民族汉族差异较大。我们需要了解, 他们的教育和成长的生命 历程中, 他或者她与其他民族个体是如何交往的。同时需要了解, 这样的交 往是基于什么交往机会或平台, 什么社会情境。这种交往给他带来的印象和 感觉是什么, 后来的发展如何? 各种短暂的、偶遇的、刻意的、长期的、频 繁的、密切的、美好的、痛苦的、甜蜜的, 等等, 这些交往的体验和感受。

每个来自民族聚居区的学生, 都有一个在其成长历程中与其他民族个体 交往的经验历程, 或者说是成长历程中的民族交往的记忆点滴。这里存在三 个值得探讨的问题。

一个是民族聚居区; 二是走出来; 三是走到哪里。研究民族交往, 选择 文化差异比较大的民族, 更有意义, 这样文化的对比较为明显。另一个方面 是, 从传统民族聚居区走出来的民族个体, 特别是与中原(内地)生活方式有 较大反差的生活环境。而民族聚居区，恰好说明某个单一民族聚居的场所， 有着较为统一的传统的单一的民族文化, 受其他文化影响较小。成长于这样 的社会环境中的某个民族个体, 是最有意义的, 最值得研究的。这是前提。 诸多经典人类学家的研究亦是如此。费孝通先生的《江村经济》跨越文野之 别, 开创了研究本民族的先例 [8], 我们这种研究不无不妥。

大多数少数民族聚居区的民族个体, 在目前的中国来说, 很少走出去。 那些能够走出来的个体, 并且与其他民族有机会长期接触交往的占据少数。 这些走出来的个体, 往往是通过做生意、工作、考学等等。我国的目前政策, 是鼓励走出去。国家政策鼓励方面有很多 [9], 比如选拔少数民族学生到内地 参加内初班、内高班、民族班; 利用少数民族优惠政策降低录取分数线录取 少数民族学生到内地读大学; 政府安排内地工厂招收少数民族工作人员 [10], 
等等。现在需要做的, 是检讨这些年以来的民族聚居区个体走出去的战略, 或者说是少数民族人口流动的问题 [11]。主要是考虑如何让这些走出民族聚居 区的民族个体能够比较顺利地融入主流社会。我们也看到了很多少数民族大 学生走了出去, 融入了主流社会。

\section{4. 结语}

促进民族交往交流需要遵循人际交往的基本规律，不能刻意地有意而为 之，不能为了交往而交往，应当创造交往的条件，交往自然而然就会发生。 进而, 民族交往需要注意文化之间的差异, 或者跨文化交往, 而不是简单地 带着刻板印象的民族身份的交往。强调民族身份的交往，容易导致民族间的 隔阂。从跨文化的个体交往的视角，避免了过于关注对方的民族身份。最后， 促进民族交往需要民族个体的社会流动，离开民族聚居区的民族个体，各民 族个体之间才有可能交往，多民族的大学就是最好的民族交往的社会场域。

\section{Conflicts of Interest}

The author declares no conflicts of interest.

\section{References}

[1] 毛胜. 坚定不移走中国特色解决民族问题的正确道路一一学习习近平关于新形 势下民族工作的重要论述 [C]//中央党史和文献研究院机构改革工作小组科研管 理组. 2016 年度文献研究个人课题成果集(上). 中共中央文献研究室科研管理部, 2018: 15 .

[2] 宗喀.漾正冈布，王振杰. 日常生活实践中的中华民族共同体构建一一以青海民 和县杏儿乡“民族团结模范集体”为例 [J]. 中南民族大学学报 (人文社会科学版), 2021, 41(8): 40-47.

[3] 辛为民. 切实把“访惠聚”活动引向深入[N]. 新疆日报(汉), 2014-08-13(001).

[4] 范崇民. “访惠聚”工作的实践与思考[N]. 新疆日报(汉), 2015-01-29(011).

[5] 孙琳娜. 大学生人际交往困扰及对策研究[J]. 公关世界, 2021(16): 61-62.

[6] 知乎. 大学里, 学习重要还是人际关系重要? [Z/OL]. https://www.zhihu.com/market/pub/19551105/manuscript/873647842135244800, 2014-11-06.

[7] 杨文娟. 跨文化交际视野下国家通用语言在新疆各族大学生文化交往中的功能 [J]. 开封教育学院学报, 2019, 39(12): 50-51.

[8] 江苏省苏州市吴江区七都镇开弦弓村志编委会. 中国名村志丛书: 开弦弓村志 [M]. 北京: 方志出版社, 2017: 44.

[9] 新华社. 第二次中央新疆工作座谈会在京召开[J]. 今日民族, 2014(6): 1-4.

[10] 陆金宝, 问亮. 新疆工会情牵内地务工少数民族职工[N]. 工人日报, 2011-04-21(002).

[11] 马戎. 民族社会学一一社会学的族群关系研究 [M]. 北京: 北京大学出版社, 2004: 557-564. 


\section{Appendix (Abstract and Keywords in Chinese)}

\section{各民族大学生交往交流机制的基础}

摘要：民族交往机制, 指的是民族交往内在的规律性以及所需要的内部和外 部的社会环境因素。在多民族大学这样的社会环境里，依据规律建立民族交 往的机制需要有三方面的基础: 首先是促进交往需要因势利导, 润物细无声。 其次是要认识到民族交往是民族个体之间的文化交往，无须刻意强调民族身 份。最后需要创造促进个体社会流动的外在社会环境。

关键词: 民族, 交往, 机制, 大学 